\title{
Maladie de Creutzfeldt-Jakob : l'intrication du gène de la PrP, d'un agent non conventionnel et de l'hormone de croissance
}

Les encéphalopathies spongiformes transmissibles sont dues à un agent non conventionnel, le prion $\left(\mathrm{m} / \mathrm{s} n^{\circ} 10\right.$, vol. 2, p. 588). Une prédisposition génétique est suspectée dans certains cas chez l'homme ( $n^{\circ} 6$, vol. 5 , p. 429 ; $n^{\circ} 1$, vol. 6, p. $77 ; n^{\circ} 3$, vol. 6, p. 311 ; $n^{\circ} 8$, vol. 6, p.813; $n^{\circ} 7$, vol. 7 , p. 754); la relation avec une mutation du gène de la protéine prion $(\operatorname{Pr} P)$ est démontrée dans les formes familiales humaines (maladie de GertsmannSträussler-Scheinker, GSS) et peut être transmise à la souris par transgénèse $\left(\mathrm{m} / \mathrm{s} n^{\circ} 2\right.$, vol. 7, p. 186). La survenue de plusieurs cas de maladie de Creutzfeldt-Jakob (MCJ) chez des enfants traités par hormone de croissance ( $m / s n^{\circ} 4$, vol. 2, p. 220), pose le problème de l'interaction entre l'hormone de croissance, l'agent pathogène véhiculé dans cet extrait hypophysaire, et les composantes génétiques de cette classe de maladie dégénérative du système nerveux central.

La maladie de Creutzfeldt-Jakob est une encéphalopathie spongiforme sporadique survenant chez l'adulte d'âge mûr (âge moyen : 60 ans). La fréquence spontanée de la maladie est estimée à un cas pour un million d'habitants [1]. C'est une démence rapidement progressive qui s'accompagne secondairement de symptômes neurologiques et, notamment, de myoclonies et d'un tracé électro-encéphalographique évocateur [2]. Le diagnostic clinique peut être confirmé par l'étude histologique du cerveau (spongiose corticale, déperdition neuronale, prolifération astrocytaire). La mise en évidence d'une transmission de la maladie au hamster ou au singe [3] par inoculation intracérébrale de tissu nerveux du sujet malade prouve le potentiel infectieux de cette maladie.

Des cas iatrogènes de maladie de Creutzfeldt-Jakob ont été rapportés depuis vingt ans [4]. L'âge moyen de ces malades est de 30 ans. Les premiers cas connus ont été transmis par un matéricl neurochirurgical insuffisamment désinfecté ou par inoculation de tissu infecté (greffes de cornée, de duremère, de tympan). Dans ces cas, l'incubation varie entre 1 et 4 ans. La transmission par de l'hormone de croissance administrée comme traitement du nanisme d'origine hypophysaire fut révélée aux États-Unis en $1985 \mathrm{~cm} / \mathrm{s}$ $n^{\circ} 4$, vol. 2, p. 220). La durée d'incubation dans les cas survenus après traitement par hormone de croissance est difficile à apprécier car le traitement dure plusieurs années. L'incubation minimale entre la fin du traitement et les premiers troubles neurologiques peut durer jusqu'à 19 ans [5]. Une quinzaine d'observations de maladie de Creutzfeldt-Jakob survenues après traitement par hormone de croissance d'origine humaine ont été publiées aux États-Unis, en Grande-Bretagne, en Nouvelle-Zélande, au Brésil et en France. L'hormone a pu constituer le vecteur de la maladie. En effet, la préparation de l'hormone de croissance consistait, jusqu'en 1988, en une extraction à partir d'hypophyses prélevées sur des cadavres. Pour fabriquer un lot d'hormone, on mélangeait ainsi 500 à 1000 hypophyses. Malgré les recommandations données pour exclure les sujets ayant présenté des troubles neurologiques, il est vraisemblable qu'en France une hypophyse au moins ait été prélevée sur un sujet porteur d'une maladie à prion. On sait que la maladie de Creutzfeldt-Jakob peut n'avoir eu aucune expression clinique alors que le système nerveux est déjà lésé de façon sévère [6] et qu'il n'existe aucun moyen actuellement de dépister cette maladie avant les premiers signes cliniques.

Les particularités cliniques des cas de maladie de Creutzfeldt-Jakob après hormone de croissance que nous avons observés à l'hôpital Trousseau (Paris, France) sont une présentation ncurologique initiale avec ataxie et diplopie, rapidement suivie d'un syndrome pyramidal, d'une rigidité extrapyramidale et d'une ophtalmoplégie [7]. Ce n'est qu'après plusieurs mois que surviennent les myoclonies, les anomalies électroencéphalographiques et la démence. Les deux premiers cas que nous avons observés sont survenus chez des enfants de 11 ans et demi et 10 ans [8].

Ces cas, qui se sont déclarés en France depuis 1989, sont survenus chez des enfants ou des adolescents. Ce sont donc les cas les plus jeunes de maladie de Creutzfeldt-Jakob décrits à notre connaissance. L'incubation est brève puisqu'elle est au maximum de 12 ans et au minimum de 18 mois chez nos patients. L'apparition à un âge précoce de ces maladies de Creutzfeldt-Jakob est difficile à expliquer. Il est possible que ces enfants aient une susceptibilité particulière à faire une maladie de Creutzfeldt-Jakob. On peut évoquer le fait que les traitements ont été commencés chez des enfants très jeunes. De ce fait, les traitements ont été très prolongés, augmentant le risque d'une contamination. Peut-être existe-t-il aussi chez le tout jeune enfant une plus grande sensibilité au prion entraînant une relativement brève incubation. Une prédisposition génétique a été envisagée. Enfin, le fait que certains de nos cas aient débuté au cours du traitement amène à s'interroger sur le rôle éventuel de l'hormone de croissance dans l'expression de la maladie.

La nature de l'agent infectant reste mystérieuse dans la maladie de Creutzfeldt-Jakob et dans les autres encéphalopathies spongiformes transmissibles connues chez l'homme (le kuru et la maladie de Gertsmann-SträusslerSheinker) et chez l'animal (la scrapie ou tremblante du mouton, la tremblante de la chèvre et la maladie des vaches folles). Cet agent infectant ne répond à aucun critère des micro-organismes connus : il résiste aux méthodes de désinfection traditionnelles et aux diverses 
méthodes de dégradation des acides nucléiques. Jusqu'à présent, aucune méthode n'a permis de mettre en évidence d'acide nucléique dans le matériel infectant purifié. Les traitements détruisant les protéines font, en revanche, disparaître le pouvoir infectant. Une protéine qui possède les caractéristiques infectieuses de cet agent non conventionnel a pu être extraite des tissus nerveux malades. Cette protéine, la $\operatorname{PrP}$, est une protéine membranaire normalement synthétisée par les neurones [9]. Elle acquiert donc un pouvoir infectant au contact de l'agent non conventionnel de l'encéphalopathie spongiforme. Les modalités de cette acquisition de virulence demeurent mystérieuses. On évoque une association de la $\operatorname{PrP}$ avec un acide nucléique de petite taille ; cette association conférerait une résistance particulière à l'ensemble qui s'accumule dans les neurones sans être détruit, et sans provoquer de réaction immunologique ni inflammatoire. L'hypothèse d'un agent protéique sans acide nucléique, le prion (proteinaceous infectious particle), a été proposée par Prusiner en 1982 [10].

A côté des maladies de CreutzfeldtJakob sporadiques et des formes iatrogènes, il existe dans environ $15 \%$ des cas, des formes familiales [11]. Ces formes rares sont marquées par une transmission verticale compatible avec une hérédité dominante. Le même mode de transmission est retrouvé dans la maladie de Gertsmann-SträusslerSheinker [12]. Dans ces deux maladies familiales ont été retrouvées des mutations sur le gène de la $\operatorname{PrP}$, considérées comme responsables de l'apparition de la maladie [13]. Dans la maladie de Gertsmann-Sträussler-Sheinker, le gène de la PrP comprend une substitution de leucine sur le codon 102 [14]. Des souris transgéniques ont été créées par introduction d'un gène de la $\operatorname{PrP}$ ayant la mutation équivalente de la maladie de Gertsmann-Sträussler-Sheinker. Ces souris transgéniques ont fait une encéphalopathie spongiforme héréditaire semblable à la maladie humaine ([15] et $m / s n^{\circ} 2$, vol. 7, p. 186). La maladie est alors plus difficile à transmettre à d'autres animaux par inoculation.

Les encéphalopathies spongiformes transmissibles ont donc une double composante : infectieuse et génétique. Les interactions entre ces deux modes de transmission ne sont pas élucidées. Chez $\mathrm{m} / \mathrm{s} n^{\circ} 6$, vol. 8, juin-juillet 92 l'homme, une éventuelle susceptibilité génétique à l'expression de la maladie est l'objet de recherches actives. En Grande-Bretagne, l'étude de la séquence du gène de la PrP de sept sujets ayant fait une maladie de Creutzfeldt-Jakob iatrogène retrouve sur le codon 129 une homozygotie $\mathrm{Val}-\mathrm{Val}$ chez quatre sujets, Met-Met chez un autre et une hétérozygotie Val-Met chez seulement deux sujets ([16] et $m / s n^{\circ} 7$, vol. 7, p. 754). La fréquence d'une homozygotie est nettement supérieure à ce que l'on rencontre dans la population générale choisie comme témoin. Toutefois, cela ne suffit pas à démontrer une susceptibilité génétique à l'infection par le prion. Des études semblables sont actuellement en cours en France et aux États-Unis.

Dans les cas que nous avons observés, l'hormone de croissance est une troisième composante à considérer. L'hormone est utilisée en thérapeutique pour ses capacités d'activation de la croissance relayée par la synthèse de facteurs de croissance (IGF). Les IGF induisent à leur tour des synthèses qui aboutissent à la croissance du cartilage de conjugaison. L'hormone de croissance et les IGF ont d'autres sites d'action, notamment au niveau du système nerveux central. Certains enfants étant encore traités au début de la maladie, un rôle propre de l'hormone de croissance dans l'apparition de la maladie de Creutzfeldt-Jakob doit être envisagé, mais reste à démontrer.

Les incertitudes concernant l'étiologie, la physiopathologie et la génétique de ces maladies constituent un extraordinaire champ de réflexion scientifique et médicale. La survenue de cas de maladie de Creutzfeldt-Jakob après traitement par hormone de croissance apporte de nouvelles données cliniques et épidémiologiques dont l'analyse doit fournir des renseignements utiles sur les interactions complexes entre l'hormone de croissance, le gène de la $\operatorname{PrP}$ et un agent pathogène non conventionnel. Pour le clinicien, cette complication dramatique rappelle à la prudence et à la modestie dans l'utilisation des thérapeutiques substitutives en pathologie humaine

\section{Thierry Billette de Villemeur} Praticien hospitalo-universitaire, neuropédiatrie, hôpital Necker-Enfants Malades, 149, rue de Sevres, 75015 Paris, France.

\section{RÉFÉRENCES}

1. Brown P, Cathala F, Raubertas RF, Gajdusek DC, Castaigne P. The epidemiology of Creutzfeldt-Jakob disease : conclusion of a 15-year investigation in France and review of the world literature: Neurology 1987; 37 : 895-904

2. Brown P, Cathala F, Castaigne P, Gajdu sek DC. Creutzfeldt-Jakob disease : clinical analysis of a consecutive series of 230 neuropathologically verified cases. Ann Neurol 1986 ; 20 : 597-602.

3. Gibbs CJ, Gajdusek DC, Asher DM, et al. Creutzfeldt-Jakob disease (spongiform encephalopathy) : transmission to chimpanzee. Science 1968 ; 161 : 388-9.

4. Duffy P, Wolf J, Collins G, De Voe AG, Streeten B, Cowen D. Possible person-to-person transmission of Creutzfeldt-Jakob disease. $N$ Engl J Med 1974 ; 290 : 692-3.

5. Marzewski DJ, Towfighi J, Harrington MG, Merril CR, Brown P. Creutzfeldt-Jakob disease following pituitary-derived human growth hormone therapy : a new American case. Neurology 1988 ; 38 : 1131-3.

6. New MI, Brown P, Temeck JW, Owens C, Hedley-Whyte ET, Richardson EP. Preclinical Creutzfeldt-Jakob disease discovered at autopsy in a human growth hormone recipient. Neurology $1988 ; 38$ : 1133-4.

7. Billette de Villemeur $T$, Gourmelen $M$, Beauvais $\mathrm{P}$, et al. Maladie de Creutzfeldt-Jakob iatrogène chez quatre enfants traités par hormone de croissance. Rev Neurol 1992 ; 148 (sous presse).

8. Billette de Villemeur T, Beauvais P, Gourmelen M, Richardet JM. Creutzfeldt-Jakob disease in children treated with growth hormone. Lancet 1991 ; 337 : 864-5.

9. Basler K, Oesch B, Scott M, et al. Scrapie and cellular $\operatorname{PrP}$ isoforms are encoded by the same chromosomal gene. Cell 1986; 46 : 417-28.

10. Prusiner SB. Novel proteinaceous infectious particles cause scrapie. Science $1982 ; 216$ : 136-44.

11. Master CL, Gajdusek DC, Gibbs CJ. The familial ocurrence of Creutzfeldt-Jakob disease and Alzeimer's disease. Brain 1981; 104 : 535-58.

12. Gerstmann J, Sträussler E, Scheinker I. Über eine eigenartige hereditär-familiäre Erkrankung des Zentralnervensystem zulgleich ein Beitrag zur Frage des vorzeitigen lokalen Alterns. $Z$ Neurol 1936 ; 154 : 736-62.

13. Hsiao K, Prusiner SB. Inherited human prion diseases. Neurology $1990 ; 40: 1820-7$.

14. Hsiao K, Baker HF, Crow TJ, et al. Linkage of a prion protein missense variant to Gerstmann-Sträussler syndrome. Nature 1989 ; $338: 342-5$

15. Hsiao KK, Scott M, Foster D, Groth DF, DeArmond J, Prusiner SB. Spontaneous neurodegeneration in transgenic mice with mutant prion protein. Science 1990 ; 250 : 1587-90.

16. Collinge J, Palmer MS, Dryden AJ. Genetic predisposition to iatrogenic Creutzfeldt-Jakob disease. Lancet $1991 ; 337$ : 1441-2. 